\title{
Performing all major surgical procedures robotically will prolong wait times for surgery
}

This article was published in the following Dove Press journal:

Robotic Surgery: Research and Reviews

17 August 2017

Number of times this article has been viewed

\author{
Brian M Shinder,** \\ Nicholas J Farber ${ }^{1, *}$ \\ Robert E Weiss ${ }^{1,2}$ \\ Thomas L Jang ${ }^{1,2}$ \\ Isaac Y Kim ${ }^{1,2}$ \\ Eric A Singer ${ }^{1,2}$ \\ Sammy E Elsamra ${ }^{1,2, *}$ \\ 'Division of Urology, Department \\ of Surgery, Rutgers Robert Wood \\ Johnson Medical School, New \\ Brunswick, NJ, USA; ${ }^{2}$ Section of \\ Urologic Oncology, Rutgers Cancer \\ Institute of New Jersey, New \\ Brunswick, NJ, USA \\ *These authors contributed equally to \\ this work
}

\begin{abstract}
This article aimed to assess the burden of scheduling major urologic oncology procedures if all cases were performed robotically and to determine whether this would increase the time a patient would have to wait for surgery. We retrospectively determined the number of prostatectomies, radical nephrectomies, partial nephrectomies, and cystectomies at a single institution for one calendar year. A hypothetical situation was then constructed where all procedures were performed robotically. Using the allotted number of days that each surgeon was able to schedule robotic procedures, we analyzed the amount of time it would take to schedule and complete all cases. Five fellowship-trained surgeons were included in the study and accounted for 317 surgical cases. Three of the surgeons had dedicated robotic surgery (RS) time (block time), while two surgeons scheduled when there was non-dedicated RS time (open time) available. If all cases were performed robotically an additional 32 days would be needed, which could significantly increase the wait time to surgery. The limited number of robotic systems available in most hospitals creates a bottleneck effect; whereby increasing the number of cases would considerably lengthen the waiting time patients have for surgery. As RS becomes increasingly more commonplace in urology and other surgical fields, this could create a significant problem for health care systems.
\end{abstract}

Keywords: surgical procedures, robotic, scheduling

\section{Introduction}

Since receiving US Food and Drug Administration approval in the year 2000, the da Vinci Surgical System (Intuitive Surgical Inc, Sunnyvale, CA, USA) has become an integral tool for urologic surgeons. In just a few years it has been widely adopted for the treatment of a variety of urologic conditions. Robotic prostatectomy has become the standard of care for men in need of prostate cancer surgery, with the vast majority of prostatectomies now performed with robotic assistance. ${ }^{1,2}$ Additionally, partial and radical nephrectomies, cystectomies, pyeloplasties, and adrenal surgeries are now routinely done robotically, especially in large tertiary care centers.

Robotic surgery (RS) offers many advantages over traditional open surgery, making it an attractive alternative to both the patient and surgeon. It was introduced to expand on the benefits of minimally invasive and laparoscopic surgical techniques, as well as reduce the difficulty in performing laparoscopic procedures, especially for laparoscopic-naïve surgeons. ${ }^{3}$ However, it is not without its pitfalls. One of the most common criticisms of RS is that the costs associated with it are much higher than that of open surgery. Various factors account for the higher cost, including the initial investment in purchasing the system, maintenance, disposable equipment fees,
Correspondence: Sammy E Elsamra Division of Urology, Department of Surgery, Rutgers Robert Wood Johnson Medical School, I Robert Wood Johnson Place, MEB Suite 584, New Brunswick, NJ 08902, USA

Tel +l 7322357775

Fax +I 7322356042

Email elsamrsa@rutgers.edu 
and longer operating room times. ${ }^{4,5}$ However, the potential clinical benefits of robotic surgery, including decreased post-operative pain, fewer blood transfusions, and shorter hospital stay make up for the increased costs of the procedure, especially at high-volume centers. ${ }^{6-8}$

As RS technology continues to advance and physicians increase the scope of surgical cases performed robotically, it is imperative to fully analyze the extent to which it can be implemented into clinical practice. In this respect, we constructed a model in which the most commonly performed urologic oncology procedures in a calendar year at a single institution were done robotically. Because the physicians are limited by the number of days allotted to them to use the robot, we hypothesized that performing all cases robotically would create a bottleneck effect, and the same caseload would not be able to be done in one calendar year.

\section{Materials and methods}

\section{Robotic console scheduling}

Our institution currently has two da Vinci robotic operating consoles available for use. They are shared among the urology (general urology, pediatric urology, and urologic oncology), gynecology (benign gynecology and gynecologic oncology), cardiothoracic, otolaryngology, general, colorectal, bariatric, and surgical oncology faculty, along with private practice physicians. Surgeons are allotted a certain number of days per month ("block time") that they are able to schedule robotic surgeries, which is determined by hospital administration based on utilization and volume. At our institution, each surgeon is only permitted to run one operating room at a time, so concurrent RS is prohibited.

\section{Data acquisition and projections}

Following Institutional Review Board approval from Rutgers Robert Wood Johnson University Hospital, a complete list of surgical cases, both open and robotic surgery, completed by five fellowship-trained urologic surgeons was generated for a single calendar year (2015). All patient data was anonymized in order to maintain patient confidentiality. This list was queried for prostatectomy, partial nephrectomy, radical nephrectomy, and cystectomy. Block times were provided by the surgeon and confirmed by the surgery scheduling coordinator. During this study period, three of the five surgeons were allotted block time, while the remaining two surgeons were only able to schedule robotic cases when there were unused or open days (open time).

Based on the average times to perform each particular surgery, we set the maximum number of robotic cases that would be able to be performed each day ("robotic days") by a surgeon with two for radical nephrectomy, partial nephrectomy, prostatectomy, and one for cystectomy. We fitted the total number of cases that a surgeon had in the study year to this scheduling framework in order to model the scenario of all surgeries being performed robotically. We then projected the number of days needed for the five surgeons to complete their caseload and whether this could be done in a year based on the allotted block times.

\section{Results}

In total, 317 major oncologic procedures were performed by the five included surgeons at a single institution in one calendar year (Table 1). Block times were 8 days per month (96 per year) for surgeon \#1, 2 days per month for surgeon \#2 (24 per year), and 2 days per month for surgeon \#3 (24 per year). Surgeon \#4 and surgeon \#5 did not have any allotted block time. Surgeon \#1 performed 126 total cases, which would require 63 robotic days to complete based on the type of surgeries performed. With 96 days per year available, there would be 33 open days. Surgeon \#2 had 42 total cases, which would take 22 robotic days to complete. This surgeon had 24 days available for the year, leaving 2 unused days. Surgeon \#3 had 46 total cases, accounting for 26 robotic days. Surgeon \#3 had only 24 days of block time, so 2 additional days would be needed to complete all the cases. Surgeons \#4 and \#5 did not have any allotted block time and performed 103 major oncologic surgeries, requiring 65 days based on the types of procedures.

Table I Surgeon caseload and block time data.

\begin{tabular}{|c|c|c|c|c|c|c|}
\hline & $\begin{array}{l}\text { Total number of } \\
\text { major oncologic } \\
\text { surgeries }\end{array}$ & $\begin{array}{l}\text { Robotic days } \\
\text { needed (numberl } \\
\text { year) }\end{array}$ & $\begin{array}{l}\text { Block time (days/ } \\
\text { month) }\end{array}$ & $\begin{array}{l}\text { Block time (days/ } \\
\text { year) }\end{array}$ & $\begin{array}{l}\text { Projected unused } \\
\text { days }\end{array}$ & $\begin{array}{l}\text { Projected extra } \\
\text { days needed }\end{array}$ \\
\hline Surgeon \# I & 126 & 63 & 8 & 96 & 33 & - \\
\hline Surgeon \#2 & 42 & 22 & 2 & 24 & 2 & - \\
\hline Surgeon \#3 & 46 & 26 & 2 & 24 & - & 2 \\
\hline Surgeon \#4 & 97 & 62 & $\mathrm{~N} / \mathrm{a}$ & $\mathrm{N} / \mathrm{a}$ & - & 62 \\
\hline Surgeon \#5 & 6 & 3 & $\mathrm{~N} / \mathrm{a}$ & $\mathrm{N} / \mathrm{a}$ & - & 3 \\
\hline
\end{tabular}

Abbreviaton: N/a, not applicable. 
In total, there would be 35 unused days by surgeon \#1 and \#2, while surgeons \#3, \#4, and \#5 would need 67 days. Scheduling permitting, an extra 32 days would then be needed for surgeons to perform the entire caseload robotically.

\section{Discussion}

The only commercially available RS platform, the da Vinci Surgical System, offers an improvement on traditional laparoscopic techniques. It employs a high-definition camera with a three-dimensional viewing system that allows up to $12 \times$ magnification. Additionally, the patented Endowrist technology mimics the user's hand dexterity, in a scaled fashion, to give 7 degrees of freedom to the operating tools, while reducing physiological tremors to allow for precision movements. ${ }^{9}$ These factors have been suggested to yield shorter learning curves, and potentially improved surgical outcomes. ${ }^{3,10}$

Over the past decade, the utilization of RS in urology has substantially increased. Some of the most complex pelvic surgeries that were almost exclusively done with open techniques in the past have been successfully performed using the da Vinci robot. Reports of level III inferior vena cava thrombectomies, appendicovesicostomy, retroperitoneal lymph node dissection, pelvic exenteration, and other complex procedures have been described in the literature. ${ }^{11-14} \mathrm{RS}$ may be well suited for these complex cases as the robotic arms eliminate the ergonomic challenges inherent in traditional laparoscopy and open surgery. ${ }^{15}$ Additionally, innovative advances in the robotic platform have allowed some to perform cases using a single port, which can minimize cosmetic defects even further. ${ }^{16}$

Although no conclusions on the true clinical benefit of RS compared to open surgery has been reached, current evidence supports its role as a prominent alternative. RS has consistently demonstrated a superior ability to limit blood loss and the amount of analgesia required for patients. ${ }^{17,18}$ Importantly, for radical cystectomy, partial nephrectomy, and radical nephrectomy, equivalent oncologic outcomes have been seen in patients undergoing robotic versus open surgery. ${ }^{9,19,20}$ On the other hand, robot-assisted radical prostatectomy may indeed provide advantageous oncologic outcomes. A recent analysis of the National Cancer Database found that RS reduced the rate of surgical margins, need for postoperative radiation therapy, and 30-day mortality compared to open surgery. ${ }^{21}$ Additionally, robotic prostatectomy has been associated with superior continence rates and sexual function following surgery. ${ }^{22,23}$ Although the costs of robotic prostatectomy are most often higher than open prostatectomy, ${ }^{4,24}$ the potential for superior outcomes could support its role as the standard of care for men with prostate cancer. ${ }^{25}$

This article highlights a potential barrier to further dissemination of RS into clinical practice. At our institution, the da Vinci robot is shared among the urology, gynecology, cardiac, ENT, general, bariatric, colorectal, and surgical oncology faculty as well as private practice surgeons. As such, most surgeons who perform robotic surgical procedures are assigned block times. Here, we demonstrate a hypothetical situation at a single institution where if all urologic procedures that could be performed robotically were done so, a bottleneck effect would have been created by the limited availability of the robot. Twenty additional days would be needed to complete the caseload from the study year, which could take several months depending on the block time available. For example, even for surgeon \#1 who had the most block time ( 8 days per month), it would take 2.5 months more for the caseload to be completed. This could potentially have significant implications for patients. In the oncologic setting, performing curative surgery with minimal delay is necessary. Indeed, extended wait times may have a detrimental effect on not just clinical outcomes, but also on a patient's anxiety and overall quality of 1 ife. ${ }^{26}$ We believe this highlights an important obstacle to further RS usage. As nearly every urology program now heavily focuses on RS training, urologic surgeons are more comfortable than ever in performing robotic procedures. Similar to how robotic-assisted radical prostatectomy has mostly overtaken open radical prostatectomy as the treatment of choice for prostate cancer, it is not difficult to imagine a scenario in which the vast majority of urologic procedures are performed robotically.

Especially in large, tertiary care academic institutions, it is expected that more complex cases will be performed robotically. Surgical innovation and early adaptation of novel and complex robotic approaches can increase the case-load being performed on these robotic systems. Recent reports of robotic cardiothoracic, ${ }^{27}$ neurosurgical ${ }^{28}$ and otolaryngologica ${ }^{29}$ procedures suggest that the da Vinci robot may also soon be routinely utilized in these fields as well. This will put even more strain on the ability of surgeons to schedule robotic procedures in a reasonable time period. Increasing the number of systems available would help eliminate this strain, though the costs of doing so are seemingly prohibitive for most organizations. Thus, creative ways to increase the availability of robotic consoles 
to surgeons must be generated. Additionally, surgeons must continue to practice and refine open and laparoscopic surgical techniques, even in the face of continued robotic innovation and especially for procedures that do not necessarily demonstrate benefit with a RS approach. Future reimbursement models, such as pay-for-performance, and other changes to the health care economy may further factor into which cases can and should be performed robotically versus open or laparoscopically.

This article describes a model showing a significant increase in the amount of time it would take to complete a year's worth of surgical cases if all cases were done robotically. This is of critical importance given a clear conflict on the horizon as the demand for RS from patients and physician training on the robotic platform rises and health care costs continue to increase unsustainably. Further, oncologic implications of performing surgery only when a robotic system is available can potentially make the difference between oncologic cure and metastatic or unresectable disease. This study, however, is not without flaws. First, it is a single institution experience, to which surgery scheduling procedures and practices may not be generalizable to others. Additionally, our model is not very sophisticated as RS feasibility is based on a myriad of factors including surgeon availability, patient availability, and robotic time availability. Further, even when all three align, factors such as patient fitness for RS (both from preoperative clearance standpoint and anatomic feasibility standpoint) will arise and preclude $100 \%$ efficiency. Our generalizations of two cases per day save for robotic radical cystectomies, and our inability to look at each individual case based on its complexity, may introduce error into this model. Certainly, a more nuanced system would include numerous other factors (non-urologic surgeons, non-urologic block time, alignment of days of week for scheduling, specific time for each procedure performed, uncommon procedures performed, etc). However, such a model would be very difficult to construct and would be accurate for only the institution studied and hence applicability to other instructions would be limited. This article does however highlight a real logistic consideration that is widely applicable to any institution that is performing $\mathrm{RS}$ at or near capacity. Further analyses should be conducted by other high-volume centers in order to fully understand the impact of increased RS utilization. While a high-quality clinical trial evaluating this topic might be difficult to execute, creating a national registry to prospectively collect information on how RS affects the length of time patients must wait to undergo an operation would be extremely valuable.

\section{Conclusion}

In summary, we described a hypothetical situation in which all prostatectomies, radical and partial nephrectomies, and cystectomies for one year were done robotically at a single institution. Based on the time available for urologists at the institution to perform robotic surgery, we projected that an extra 32 days would be needed, significantly increasing the waiting time for patients undergoing such operations. As advancements in the field continue, and more surgeons from other specialties begin to integrate RS into clinical practice, the scheduling burden can be expected to increase further. Additional research will be needed to come up with viable solutions to this problem, including evaluating the true clinical utility of robotic surgery.

\section{Acknowledgment}

This work is supported by a grant from the National Cancer Institute (P30CA072720).

\section{Disclosure}

The authors report no conflicts of interest in this work.

\section{References}

1. Cooperberg MR, Broering JM, Carroll PR. Time trends and local variation in primary treatment of localized prostate cancer. J Clin Oncol. 2010;28(7):1117-1123.

2. Ramirez D, Zargar H, Caputo P, Kaouk JH. Robotic-assisted laparoscopic prostatectomy: An update on functional and oncologic outcomes, techniques, and advancements in technology. J Surg Oncol. 2015;112(7): 746-752.

3. Giri S, Sarkar DK. Current status of robotic surgery. Indian J Surg. 2012;74(3):242-247.

4. Faiena I, Dombrovskiy VY, Modi PK, et al. Regional Cost Variations of Robot-Assisted Radical Prostatectomy Compared With Open Radical Prostatectomy. Clin Genitourin Cancer. 2015;13(5):447-452.

5. Turchetti G, Palla I, Pierotti F, Cuschieri A. Economic evaluation of da Vinci-assisted robotic surgery: a systematic review. Surg Endosc. 2012;26(3):598-606.

6. Peyronnet B, Seisen T, Oger E, et al. Comparison of 1800 Robotic and Open Partial Nephrectomies for Renal Tumors. Ann Surg Oncol. 2016;23(13):4277-4283.

7. Yaxley JW, Coughlin GD, Chambers SK, et al. Robot-assisted laparoscopic prostatectomy versus open radical retropubic prostatectomy: early outcomes from a randomised controlled phase 3 study. Lancet. 2016;388(10049):1057-1066.

8. Hu JC, Chughtai B, O'Malley P, et al. Perioperative Outcomes, Health Care Costs, and Survival After Robotic-assisted Versus Open Radical Cystectomy: A National Comparative Effectiveness Study. Eur Urol. 2016;70(1):195-202.

9. Ficarra V, Cavalleri S, Novara G, Aragona M, Artibani W. Evidence from robot-assisted laparoscopic radical prostatectomy: a systematic review. Eur Urol. 2007;51(1):45-55; discussion 56.

10. Ahlering TE, Skarecky D, Lee D, Clayman RV. Successful transfer of open surgical skills to a laparoscopic environment using a robotic interface: initial experience with laparoscopic radical prostatectomy. $J$ Urol. 2003;170(5):1738-1741.

11. Gill IS, Metcalfe C, Abreu A, et al. Robotic Level III Inferior Vena Cava Tumor Thrombectomy: Initial Series. J Urol. 2015;194(4):929-938. 
12. Chung PH, De S, Gargollo PC. Robotic appendicovesicostomy revision in children: description of technique and initial results. $J$ Endourol. 2015;29(3):271-275.

13. Dudderidge T. Retroperitoneal lymph node dissection (RPLND) open surgery's next challenger is ready to enter the ring. BJU Int. 2015;115(1):3-4.

14. Castillo OA, Vidal-Mora I, Rodriguez-Carlin A, Silva A, Schatloff O. First Report: Robot-Assisted Total Pelvic Exenteration for Locally Advanced Prostate Cancer. J Laparoendosc Adv Surg Tech A. 2015;25(7): 592-594.

15. Hemal AK, Menon M. Robotics in urology. Curr Opin Urol. 2004;14(2):89-93.

16. Kaouk JH, Goel RK, Haber GP, Crouzet S, Stein RJ. Robotic single-port transumbilical surgery in humans: initial report. BJU Int. 2009;103(3):366-369.

17. Parekh DJ, Messer J, Fitzgerald J, Ercole B, Svatek R. Perioperative outcomes and oncologic efficacy from a pilot prospective randomized clinical trial of open versus robotic assisted radical cystectomy. $J$ Urol. 2013;189(2):474-479.

18. Ficarra V, Minervini A, Antonelli A, et al. A multicentre matched-pair analysis comparing robot-assisted versus open partial nephrectomy. BJU Int. 2014;113(6):936-941.

19. Snow-Lisy DC, Campbell SC, Gill IS, et al. Robotic and laparoscopic radical cystectomy for bladder cancer: long-term oncologic outcomes. Eur Urol. 2014;65(1):193-200.

20. Minervini A, Vittori G, Antonelli A, et al. Open versus robotic-assisted partial nephrectomy: a multicenter comparison study of perioperative results and complications. World J Urol. 2014;32(1):287-293.
21. Pearce SM, Pariser JJ, Karrison T, Patel SG, Eggener SE. Comparison of Perioperative and Early Oncologic Outcomes between Open and Robotic Assisted Laparoscopic Prostatectomy in a Contemporary Population Based Cohort. J Urol. 2016;196(1):76-81.

22. O’Neil B, Koyama T, Alvarez J, et al. The Comparative Harms of Open and Robotic Prostatectomy in Population Based Samples. J Urol. 2016;195(2):321-329.

23. Haglind E, Carlsson S, Stranne J, et al. Urinary Incontinence and Erectile Dysfunction After Robotic Versus Open Radical Prostatectomy: A Prospective, Controlled, Nonrandomised Trial. Eur Urol. 2015;68(2):216-225.

24. Leow JJ, Chang SL, Meyer CP, et al. Robot-assisted Versus Open Radical Prostatectomy: A Contemporary Analysis of an All-payer Discharge Database. Eur Urol. 2016;70(5):837-845.

25. Koch MO. Robotic versus Open Prostatectomy: End of the Controversy. J Urol. 2016;196(1):9-10.

26. Bourgade V, Drouin SJ, Yates DR, et al. Impact of the length of time between diagnosis and surgical removal of urologic neoplasms on survival. World J Urol. 2014;32(2):475-479.

27. Broussard BL, Wei B, Cerfolio RJ. Robotic surgery for posterior mediastinal pathology. Ann Cardiothorac Surg. 2016;5(1):62-64.

28. Marcus HJ, Hughes-Hallett A, Cundy TP, Yang GZ, Darzi A, Nandi D. da Vinci robot-assisted keyhole neurosurgery: a cadaver study on feasibility and safety. Neurosurg Rev. 2015;38(2):367-371; discussion 371.

29. Gungadeen A, Lisseter R, Manickavasagam J, Paleri V. Transoral robotic resection of a large schwannoma in the retropharyngeal space. J Laryngol Otol. 2016;130(4):401-403.
Robotic Surgery: Research and Reviews

\section{Publish your work in this journal}

Robotic Surgery: Research and Reviews is an international, peer reviewed open access, online journal publishing original research, commentaries, reports, and reviews on the theory, use and application of robotics in surgical interventions. Articles on the use of supervisory-controlled robotic systems, telesurgical devices, and shared-control systems are

\section{Dovepress}

invited. The manuscript management system is completely online and includes a very quick and fair peer review system, which is all easy to use. Visit http://www.dovepress.com/testimonials.php to read real quotes from published authors. 